\title{
Leitthema
}

Orthopäde 2019 • 48:776-783

https://doi.org/10.1007/s00132-019-03790-x

Online publiziert: 8. August 2019

(c) Der/die Autor(en) 2019

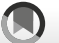

Check for
updates

S. Scheipl' - B. Liegl-Atzwanger ${ }^{2}$ - J. Szkandera ${ }^{3}$ B. Rinner ${ }^{4}$ C. Viertler ${ }^{2}$.

J. Friesenbichler ${ }^{1}$ M. Bergovec ${ }^{1} \cdot$ A. Leithner ${ }^{1}$

'Univ.-Klinik für Orthopädie und Traumatologie, Medizinische Universität Graz, Graz, Österreich

${ }^{2}$ Diagnostik- und Forschungsinstitut für Pathologie, Medizinische Universität Graz, Graz, Österreich

${ }^{3}$ Univ.-Klinik für Innere Medizin, Klin. Abt. für Onkologie, Medizinische Universität Graz, Graz, Österreich

${ }^{4}$ Biomedizinische Forschung, Medizinische Universität Graz, Graz, Österreich

\section{Gibt es eine Magic Bullet für Sarkome?}

\section{Personalisierte Therapie von malignen Tumoren des Stütz- und Bewegungsapparates}

\begin{abstract}
Personalisierte Therapien von Tumorerkrankungen zielen darauf $a b$, spezifische überlebenswichtige Mechanismen eines Tumors bei bestmöglicher Schonung des restlichen Organismus medikamentös zu unterbinden. Maßgeschneiderte Therapeutika sind mittlerweile für viele Tumorerkrankungen im Einsatz. Damit stellt sich die Frage, inwieweit diese neuen Therapieformen auch für maligne Tumoren des Stütz- und Bewegungsapparates relevant werden.
\end{abstract}

\section{Personalisierte Behandlung von Tumorerkrankungen}

Das Ziel einer personalisierten Krebsbehandlung ist es, auf zellulärer Ebene tumorspezifische Entstehungs- und Überlebensmechanismen zu identifizieren, welche die Entwicklungen von "maßgeschneiderten“ Behandlungsstrategien erlauben. Durch diese soll ein optimaler, gegen den Tumor gerichteter Behandlungseffekt erzielt und der restliche Organismus weitgehend geschont werden [29].

\section{„Wir müssen chemisch zielen lernen"}

Dieses Konzept ist keineswegs neu und geht auf den deutschen Medizi- ner und Nobelpreisträger Paul Ehrlich (1854-1915) zurück [27, 29]: Basierend auf seinen Beobachtungen im Rahmen der Behandlung von Infektionskrankheiten erkannte er, dass diese Therapien effizienter und besser verträglich waren, sofern sie sich gegen Strukturen richteten, die ausschließlich in den eindringenden Parasiten bzw. Keimen vorkamen, nicht jedoch im Wirtsorganismus. Daraus entwickelte er jene den maßgeschneiderten Therapiekonzepten zugrundeliegende Vision einer "Zauberkugel“ (englisch: "magic bullet"), welche sich analog zu einer Gewehrkugel selektiv gegen Krankheitserreger richtet, gesundes Gewebe jedoch schont $[27,29]$.

\section{Anfänge der maßgeschneiderten Krebstherapie}

Knapp 100 Jahre später wurde diese Idee wieder aufgegriffen und erhielt neue Nahrung: So wurde der Biochemiker Stanley Cohen im Jahr 1986 mit dem Nobelpreis für Physiologie und Medizin ausgezeichnet. Er erhielt diesen für die Beschreibung des sogenannten epidermalen Wachstumsfaktors („epidermal growth factor", EGF) und des dazugehörigen Rezeptors an der Zelloberfläche (,epidermal growth factor receptor", EGFR). Dieses System von Ligand und Rezeptor funktioniert nach einem Schlüssel-Schloss-Prinzip und

\begin{tabular}{|c|c|}
\hline \multicolumn{2}{|c|}{ Abkürzungen } \\
\hline$A B L$ & „Abelson tyrosine kinase" \\
\hline$B C R$ & „Breakpoint cluster region" \\
\hline$C D$ & "Cluster of differentiation" \\
\hline$c-K I T$ & $\begin{array}{l}\text { "Stem-cell factor receptor"; } \text {, } \text {, receptor } \\
\text { tyrosine kinase CD } 117 \text { " }\end{array}$ \\
\hline COL1A1 & "Collagen type I alpha 1 chain" \\
\hline CSF & "Colony stimulating factor" \\
\hline DFSP & Dermatofibrosarcoma protuberans \\
\hline$E G F$ & „Epidermal growth factor" \\
\hline EGFR & „Epidermal growth factor receptor" \\
\hline ETV6 & „ETS variant transcription factor 6" \\
\hline EWSR1 & „Ewing sarcoma breakpoint region“ 1 \\
\hline$F D A$ & Food and Drug Administration \\
\hline FLI1 & "Friend leukemia virus integration" 1 \\
\hline GIST & Gastrointestinaler Stromatumor \\
\hline HER2 & $\begin{array}{l}\text { „Human epidermal growth factor } \\
\text { receptor" } 2\end{array}$ \\
\hline$I D H$ & Isozitratdehydrogenase \\
\hline NTRK & $\begin{array}{l}\text { "Neurotrophic receptor tyrosin } \\
\text { kinase" }\end{array}$ \\
\hline PDGFB & $\begin{array}{l}\text { "Platelet-derived growth factor beta } \\
\text { polypeptide" }\end{array}$ \\
\hline PDGFR & $\begin{array}{l}\text { "Platelet-derived growth factor } \\
\text { receptor" }\end{array}$ \\
\hline RANKL & „Receptor activator of NF-kB ligand" \\
\hline SFT & Solitärer fibröser Tumor \\
\hline$T K I$ & Tyrosinkinaseinhibitor \\
\hline
\end{tabular}




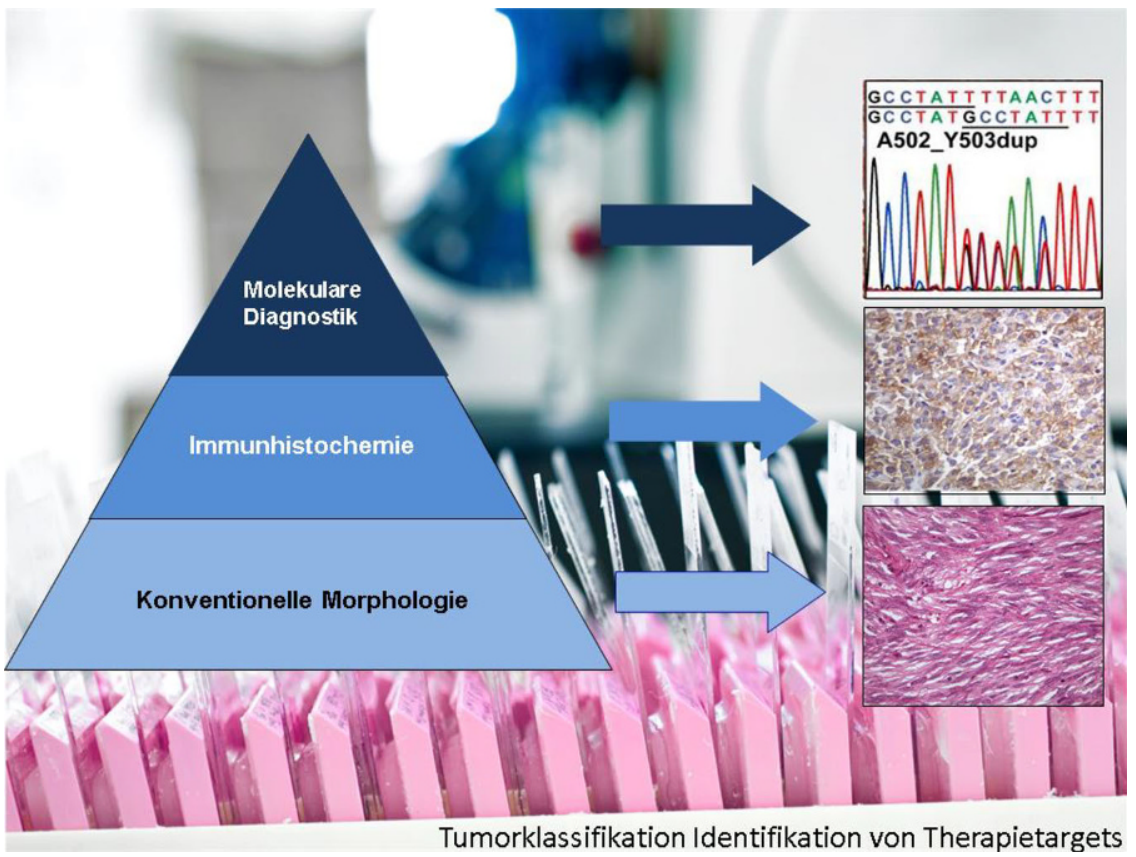

Abb. $1 \Delta$ Algorithmus zur pathologischen Tumordiagnostik. (Mit freundl. Genehmigung, ๑ B. Liegl-Atzwanger, alle Rechte vorbehalten)

steuert unter anderem das Wachstum der Zelle [27, 29]. Diese Entdeckung war wegbereitend für die Entwicklung von maßgeschneiderten Tumortherapien. Im Jahr 1998 wurde mit Trastuzumab (Herceptin ${ }^{\circledR}$, Roche/Genentech Inc., Basel, Schweiz) der erste humanisierte monoklonale Antikörper durch die USamerikanische Food and Drug Administration (FDA) zugelassen, welcher sich gegen ERBB2 (besser bekannt als HER2/neu) richtet. Dies ist ein Rezeptor für Wachstumsfaktoren, welcher auf Burstkrebszellen vielfach vermehrt anzutreffen ist. Trastuzumab wird nach wie vor erfolgreich in der Behandlung von HER2/neu-positivem Brustkrebs eingesetzt [29].

Auf Paul Ehrlich geht auch die Idee zurück, eine solche „Zauberkugel“ durch die systematische chemische Weiterentwicklung von Substanzen zu erzeugen, um deren Treffsicherheit gegen Pathogene zu erhöhen („Wir müssen chemisch zielen lernen“) [29]. Imatinib (Glivec ${ }^{\circledR} /$ Gleevec $^{\circledR}$, Novartis Pharma AG, Basel, Schweiz) ist ein Beispiel für einen kleinmolekularen Kinaseinhibitor, welcher gezielt gegen eine krankheitsauslösende molekulare Struktur entwickelt wurde [12]. Imatinib hemmt die Tyrosinkinase BCR-ABL, welche als Genprodukt der
Philadelphia-Chromosom-Translokation $\mathrm{t}(9 ; 22)$ im Rahmen der chronischmyeloischen Leukämie entsteht. Das BCR-ABL Fusionsonkoprotein fungiert dabei als dysreguliertes, hochaktives Enzym, welches das Wachstum und die Teilung der Zellen durch seine Interaktion mit zahlreichen nachgeschalteten Signalwegen fördert und dadurch die Leukämieentstehung bewirkt [12]. Basierend auf Paul Ehrlichs Aussage „Wir müssen chemisch zielen lernen" war Imatinibmesylat der erste rational konzipierte kleinmolekulare Inhibitor, der entwickelt wurde, um selektiv die Tumorzellen, nicht jedoch die "normalen“ Zellen im Rahmen einer chronischmyeloischen Leukämieerkrankung $\mathrm{zu}$ hemmen. Aus diesem Grund gilt Imatinib als Paradigma der maßgeschneiderten Krebstherapie (englisch: „targeted therapy“) [29].

\section{Neue Erkenntnisse erweitern die Diagnostik und Therapie}

Maligne Tumoren des Knochens und der Weichteile („Sarkome“) sind ausgesprochen seltene Tumoren (sogenannte „orphan diseases“). Ihre Inzidenz beträgt 4-5/100.000/Jahr, die Inzidenz der Knochensarkome liegt noch darunter $[6,7]$.
Ein multidisziplinärer therapeutischer Ansatz ist für diese Tumoren obligat [6, 7, 19]. Neben der lokalen Therapie mittels weiter/radikaler chirurgischer Resektion und (neo-)adjuvanter Bestrahlungsbehandlung galten Chemotherapien, insbesondere solche auf DoxorubicinBasis, lange Zeit als ausschließliche systemische Behandlungsoptionen für viele Knochen- und auch Weichteilsarkome $[6,7,19]$. Mit dem Aufkommen der molekularpathologischen Diagnostik wurden bei vielen Sarkomen charakteristische genetische Veränderungen beschrieben, sodass die Molekularpathologie aus der Routinediagnostik von Sarkomen nicht mehr wegzudenken ist (• Abb. $1 ;[6,7$, 19, 20]). Die molekulare Tumorklassifikation unterscheidet dabei komplexe genetische Veränderungen, welche beispielsweise bei Osteosarkomen oder Chordomen anzutreffen sind, von „einfachen " genetischen Veränderungen wie Translokationen, Amplifikationen, oder Mutationen. Speziell letztere stellen potenzielle therapeutische Targets dar [6, 7, 19, 20]. Basierend auf diesen Erkenntnissen hat die Entwicklung von personalisierten Therapien auch den Bereich muskuloskelettaler Tumoren voll erfasst [19]. Speziell diverse Tyrosinkinaseinhibitoren, aber auch monoklonale Antikörper, werden mittlerweile in der Therapie von Tumoren des Stütz- und Bewegungsapparates, insbesondere der Weichteile, evaluiert [19]. Demgegenüber haben immuntherapeutische Konzepte, mit wenigen Ausnahmen [8, 14], in der Sarkomtherapie aktuell noch einen untergeordneten Stellenwert [19]. Die nachfolgenden Abschnitte sollen einen Überblick über relevante genetische Veränderungen sowie aktuelle Ansätze in der personalisierten Behandlung maligner bzw. lokal aggressiver Tumoren des Stütz- und Bewegungsapparates liefern.

\section{Maßgeschneiderte Therapien für Knochensarkome?}

Als häufigste maligne Primärtumoren des Knochens sind bei Kindern und Jugendlichen das Osteo- und das EwingSarkom anzuführen [7], im höheren Erwachsenenalter das Chondrosarkom $[7,15]$. 
Orthopäde 2019 · 48:776-783 https://doi.org/10.1007/s00132-019-03790-x

(c) Der/die Autor(en) 2019

\section{S. Scheipl · B. Liegl-Atzwanger · J. Szkandera · B. Rinner · C. Viertler · J. Friesenbichler · M. Bergovec · A. Leithner}

\section{Gibt es eine Magic Bullet für Sarkome? Personalisierte Therapie von malignen Tumoren des Stütz- und Bewegungsapparates}

\section{Zusammenfassung}

\section{Hintergrund. Personalisierte Therapien} von Tumorerkrankungen zielen darauf ab, spezifische überlebenswichtige Mechanismen eines Tumors medikamentös zu unterbinden. Ziel der Arbeit und Methode. Der Beitrag soll einen Überblick über relevante genetische Veränderungen und potenzielle therapeutische Zielstrukturen von Knochenund Weichteilsarkomen vermitteln und aktuelle Ansätze in der personalisierten Behandlung maligner Tumoren des Stütz- und Bewegungsapparates darlegen.

Ergebnisse. Die molekularpathologische Diagnostik ist für Knochen- und Weichteiltumoren mittlerweile unerlässlich. Für Knochensarkome existieren noch keine rou- tinemäßig einsetzbaren maßgeschneiderten Therapeutika. Denosumab ist lediglich als symptomatische Behandlung von Riesenzelltumoren zu werten. Imatinib stellt eine maßgeschneiderte Therapie für Untergruppen maligner gastrointestinaler Stromatumoren (GIST) bzw. für Formen des Dermatofibrosarcoma protuberans dar. Der Einsatz von antiangiogenetischen Multikinaseinhibitoren und anderen Tyrosinkinaseinhibitoren (TKI) bzw. monoklonalen Antikörpern wird bei diversen Weichteilsarkomen evaluiert. Sorafenib zeigte vielversprechende Ergebnisse bei der Behandlung aggressiver Fibromatosen. Für histologiespezifische Chemotherapien konnte eine prospektiv-randomisierte Studie keinen Benefit zeigen.

Diskussion. Ein besseres Verständnis der Auswirkungen molekularpathologischer Veränderungen auf die Tumorentstehung ist vielfach noch nötig, um treffsichere Behandlungen entwickeln zu können. Klinische Studienansätze müssen effizienter werden und der Seltenheit bzw. der zunehmenden Subtypisierung von Sarkomen Rechnung tragen.

\section{Schlüsselwörter}

Medikamentenresistenz - Targeted Therapy · Monoklonale Antikörper · Osteosarkome · Tyrosinkinaseinhibitor

\section{Is there a magic bullet for sarcomas? Personalised treatment for maligant tumours of bone and soft tissue}

Abstract

Background. Personalised tumour therapies aim to selectively target pathways and structures to which a tumour shows an oncogenic addiction.

Objective and Method. This article aims to provide an overview of relevant genetic alterations in bone and soft-tissue tumours, which might serve as potential therapeutic targets for personalised medicines in the future. Recent approaches towards a personalised treatment of various tumours of bone and soft tissues are reviewed. Results. Molecular diagnosis has become an essential tool for the characterisation of bone and soft-tissue tumours. Currently, no targeted therapies are routinely available for bone sarcomas. Denosumab is merely a symptomatic treatment for giant cell tumours of the bone. Imatinib has become the paradigm of a targeted treatment for subgroups of malignant gastrointestinal stromal tumours (GISTs) and dermatofibrosarcoma protuberans. Antiangiogenic multikinase inhibitors, various other tyrosine kinase inhibitors (TKIs) and monoclonal antibodies are currently being evaluated in several (sub-) types of soft-tissue sarcomas. Sorafenib showed promising results in the treatment of aggressive desmoid-type fibromatosis. Histology-tailored chemotherapies did not yield superior results in a prospective randomised multicentre trial.

Conclusion. More in-depth knowledge is required for many sarcomas to link their genetic alterations to tumorigenesis in order to develop efficient personalised treatment strategies. Clinical trial designs need to be adapted to evaluate new therapeutic strategies in these ultra-rare tumours and their various sub-types more efficaciously.

\section{Keywords}

Drug resistance - Molecular targeted therapy . Monoclonal antibodies - Osteosarcoma . Tyrosine kinase inhibitor

\section{Ewing-Sarkome zeigen charakteristische Translokationen}

Sowohl Osteo- als auch Ewing-Sarkomen ist ein Ansprechen auf Kombinationschemotherapien gemeinsam. Sie werden im Rahmen von multizentrischen, randomisierten Studien laufend optimiert, und haben in den vergangenen Jahrzehnten als Bestandteil multimodaler Therapiekonzepte zu einer signifikanten Steigerung des Überlebens beigetragen [7]. Demgegenüber konnten mit personalisierten Therapieansätzen bis jetzt noch keine nachhaltigen Erfolge bei diesen ossären Sarkomen erzielt werden [19]. Während klassische Osteosarkome sehr heterogene genetische Veränderungen aufweisen, welche einen einheitlichen "driver", also eine definierte genetische Ursache, nach aktuellem Erkenntnisstand vermissen lassen $[7,16]$, zeichnen sich Ewing-Sarkome durch charakteristische Chromosomentranslokationen aus (• Abb. 2; [7]). Als "klassische“ und am häufigsten (in circa $85 \%$ ) beobachtete Fusion gilt $\mathrm{t}(11 ; 22)$ (q24:q12) mit den Fusionspartnern EWSR1 und FLI1, welche zur Entstehung des EWSR1-FLI1-Onkoproteins führt $[19,20]$. Dieses agiert als abnormer Transkriptionsfaktor und trägt dadurch zur Tumorentstehung bei $[19,20]$. Charakteristische Translokationen unterstützen die Entwicklung von gezielten, minimal-invasiven Diagnoseund Tumormonitoringverfahren, wie der „liquid biopsy“. Dabei gibt der Nachweis von Tumormarkern im Blut der Patientinnen und Patienten einen Hinweis auf die aktuelle Tumorlast und damit auf das Ansprechen von Therapien bzw. auf ein mögliches Wiederauftreten dieser 


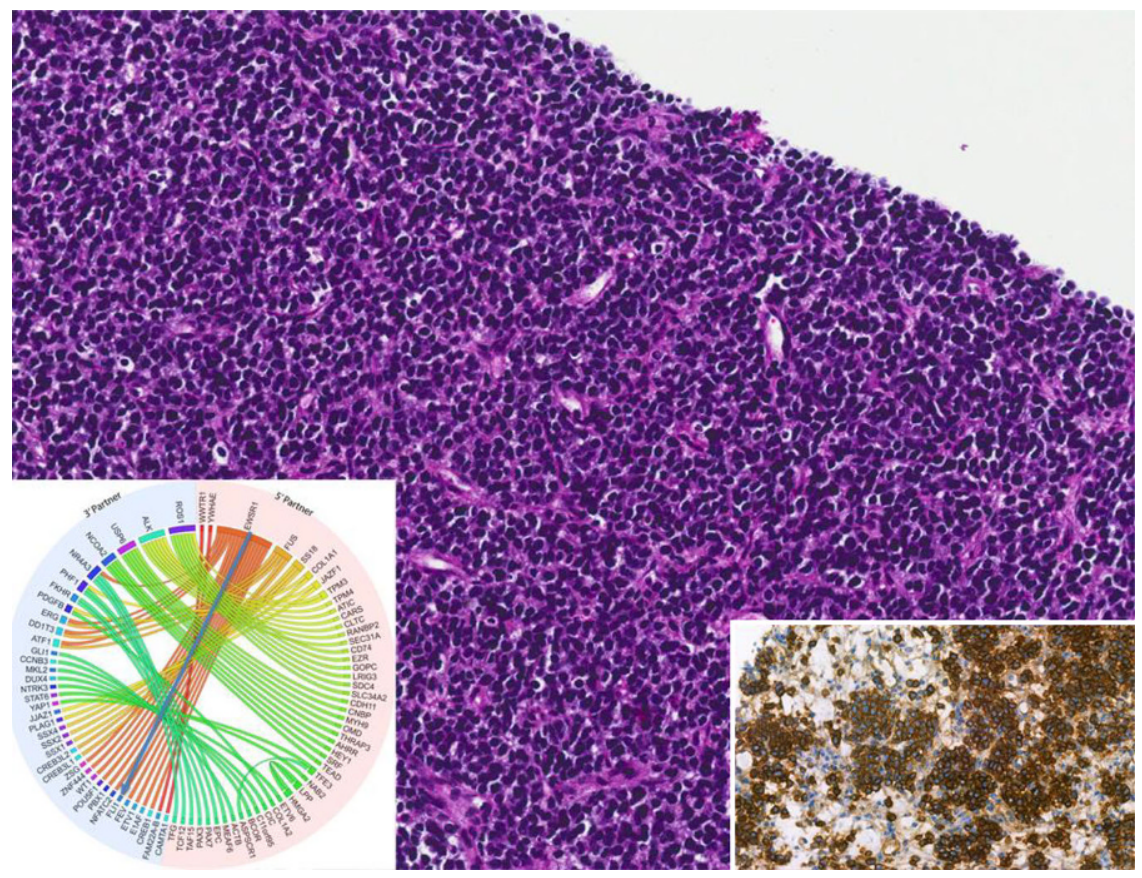

Abb. 2 A Ewing-Sarkom. Morphologie:klein-, rund-, blauzellig.Immunhistochemie:starke Positivität für CD99 (cluster of differentiation 99). Next Generation Sequencing: Nachweis der charakteristischen Fusion EWSR1-FLI1 ("Ewing sarcoma breakpoint region“ 1 bzw. „Friend leukemia virus integration" 1). (Mit freundl. Genehmigung, @ B. Liegl-Atzwanger, alle Rechte vorbehalten)

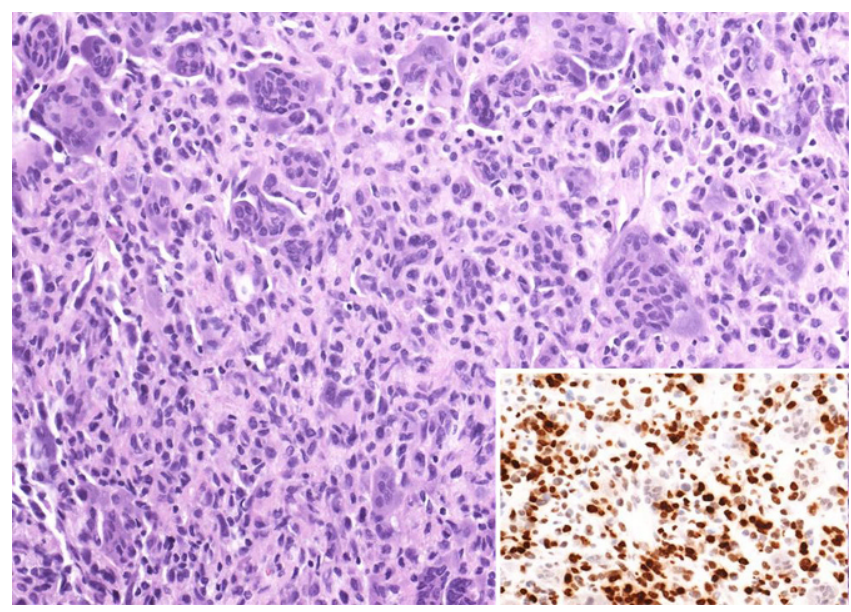

Abb. $3 \Delta$ Riesenzelltumor des Knochens mit einem rund-ovalären bis spindelzelligen, mononukleären stromalen Tumorzellproliferat und zahlreichen, untermischten osteoklastären Riesenzellen (Hämatoxylin-Eosin, 200x). Die Immunhistochemie für H3.3 (Insert) zeigt eine positive Kernreaktion der mononukleären Tumorzellen und eine negative (bzw. schwache, unspezifische) Reaktion der osteoklastären Riesenzellen. (Mit freundl. Genehmigung, @ C. Viertler, alle Rechte vorbehalten)

Tumoren [17]. Demgegenüber ist eine gezielte Entwicklung eines Inhibitors gegen das Ewing-Translokationsprodukt bei Ewing-Sarkomen bislang noch nicht gelungen $[7,20,24]$, wenngleich die präklinische Forschung dahingehende Ansätze nachdrücklich verfolgt $[9,30]$; erste kleinmolekulare Inhibitoren be-

finden sich aktuell bereits in klinischer

\section{IDH-Mutationen charakterisieren Chondrosarkome} Testung (NCT02657005) $[9,30]$.
Isozitratdehydrogenase (IDH 1 bzw. 2) codieren [15]. In der Folge führt dies zur Akkumulation des onkogenen Metaboliten (D)-2-Hydroxyglutarat, welcher den Zellstoffwechsel fundamental beeinträchtigt [22]. Bisher ist es noch nicht gelungen, den genauen Mechanismus der durch diese Mutation hervorgerufenen Störung in der Entwicklung des Knorpelgewebes hin zum Tumor zu entschlüsseln bzw. einen zuverlässigen Inhibitor gegen das fehlgeleitete Enzym und/oder den pathogenen Metaboliten $\mathrm{zu}$ identifizieren $[15,22]$.

\section{Denosumab kann Riesenzell- tumoren nicht heilen}

Ähnliches gilt für H3.3-Mutationen bei Riesenzelltumoren des Knochens und Chondroblastomen. Beide Tumorentitäten tragen in über $90 \%$ spezifische Mutationen in Genen, welche für ein Histon-Protein codieren (H3F3A bei Riesenzelltumoren und $H 3 F 3 B$ bei Chondroblastomen) [4]. Analog zu EwingSarkomen oder Chondrosarkomen ist der exakte Wirkmechanismus dieser genetischen Veränderung noch nicht identifiziert und es existiert noch kein spezifisches Therapeutikum [4, 7]. Gerade Riesenzelltumoren des Knochens, welche grundsätzlich den benignen Tumoren zugerechnet werden, können ein ausgesprochen aggressives lokales Wachstum zeigen [7]. Diese tumorbedingte Knochenresorption wird durch Osteoklasten hervorgerufen, die als namensgebende prominente Zellpopulation in diesem Tumor zu beobachten sind [7]. Eine Hemmung der Osteoklasten durch den RANKL(,receptor activator of NF-кB ligand“; "nuclear factorB ligand")-Antagonisten Denosumab ist jedoch lediglich als symptomatische Therapie anzusehen [4]. Durch die Beschreibung der Histonmutation $\mathrm{H} 3 \mathrm{~F} 3 \mathrm{~A}$ wurde bestätigt, dass nicht die osteoklastären Riesenzellen, sondern die rund-ovalären bis spindelzellförmigen, mononukleären stromalen Zellen diese Mutation aufweisen und damit die neoplastische Zellpopulation dieses Tumors darstellen (• Abb. 3; [4]). Eine Therapie mit Denosumab muss somit langfristig aufrechterhalten werden, da andernfalls
Die Mehrzahl der Chondrosarkome zeichnet sich durch Mutationen in Genen aus, welche für ein Enzym namens 

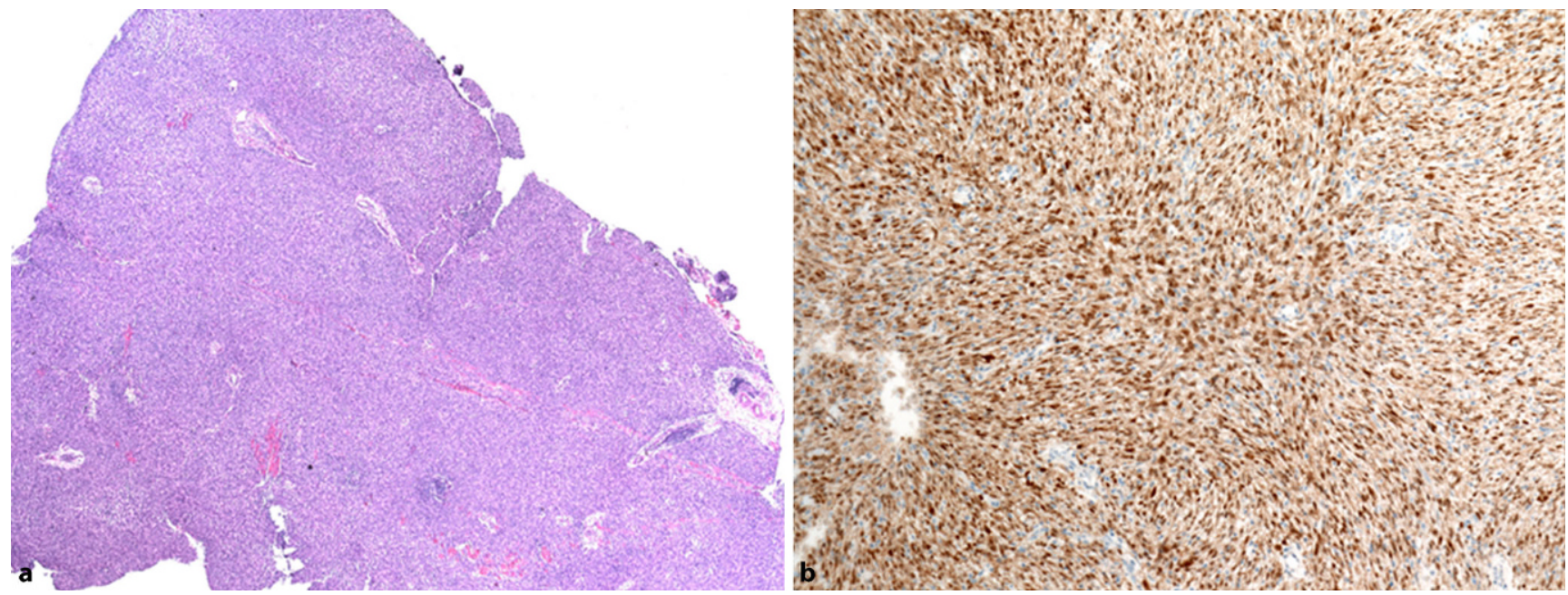

Abb. 4 ム Infantiles Fibrosarkom bei einer 5-jährigen Patientin (a). Die Immunhistochemie mit Antikörpern gegen NTRK („neurotrophic receptor tyrosin kinase") zeigt ein typisches nukleäres und zytoplasmatisches Färbemuster (b). Next Generation Sequencing bestätigt das Vorliegen einer charakteristischen NTRK3-ETV6 („ETS variant transcription factor 6")-Fusion (nicht illustriert). (Mit freundl. Genehmigung, ๑ B. Liegl-Atzwanger, alle Rechte vorbehalten)

mit einem Wiederauftreten der Symptomatik zu rechnen ist [7]. Aus diesem Grund sowie aufgrund von beobachteten bzw. vermuteten (Langzeit-)Nebenwirkungen wird Denosumab heute nur in gezielten Indikationen, zum Beispiel zur besseren präoperativen Abgrenzung bzw. bei inoperablen Fällen, verabreicht [7].

\section{Personalisierte Therapien von Weichteiltumoren}

\section{Imatinib als Pionier der personalisierten Medizin}

In den vergangenen Jahren konnten laufend neue personalisierte Therapeutika in diverse Behandlungsschemata von Weichteiltumoren integriert werden [6, 19]. Ein Pionier für einen behördlich zugelassenen, kleinmolekularen Kinaseinhibitor mit erfolgreicher Anwendung in der Therapie von Weichteiltumoren ist Imatinib [19]. Imatinib wird nicht nur in der Behandlung der chronisch-myeloischen Leukämie eingesetzt (s. oben), sondern auch in der Behandlung von soliden Tumoren. Dies ist der Tatsache geschuldet, dass Imatinibmesylat, abgesehen von BCR/ABL, auch Rezeptorkinasen an der Zelloberfläche hemmt, nämlich die Thrombozytenwachstumsfaktorrezeptoren $\alpha$ und $\beta$ (PDGFR- $\alpha$ und $-\beta$; „platelet-derived growth factor receptor" $\alpha$ und $\beta$ ), wie auch den Stamm- zellfaktorrezeptor c-KIT (CD117), und andere mehr [12]. Daher findet Imatinib Anwendung in der Therapie von KIT-mutierten, metastasierten sowie intermediate- bzw. „high-risk“ gastrointestinalen Stromatumoren (GIST) [6, 19], wie auch von Tumoren, welche eine dysregulierte PDGFR- $\alpha / \beta$ Aktivität zeigen. Dazu zählen beispielsweise eine Untergruppe von PDGFR- $\alpha$ mutierten GIST, oder auch Weichteiltumoren aus der Gruppe „Dermatofibrosarcoma protuberans" (DFSP). Diese weisen als Folge einer Translokation eine vermehrte PDGF(R)-B-Expression auf (• Abb. 4); für sie stellt die Gabe von Imatinib mittlerweile eine Standardtherapie dar [6, 19].

\section{Multikinasehemmer bei Weichteilsarkomen}

Ein weiterer Fokus in der Behandlung von Weichteilsarkomen liegt auf dem Einsatz von antiangiogenetischen Multikinaseinhibitoren, wie beispielsweise Pazopanib (Votrient ${ }^{\circledR}$, GSK, London, UK), Sorafenib (Nexavar ${ }^{\circledR}$, Bayer AG, Leverkusen, Deutschland und Onxy Pharmaceuticals, South San Francisco, CA, USA), Sunitinib (Sutent ${ }^{\circledR}$, Pfizer, New York, NY, USA) oder Regorafenib (Stivarga ${ }^{\circledR}$, Bayer). Wie die Bezeichnung bereits andeutet, hemmen Multikinaseinhibitoren eine Vielzahl von Tyrosin- kinasen [19]. Sie werden unter anderem in der Behandlung von Imatinib-resistenten GIST (Sunitinib, Regorafenib) bzw. bei diversen fortgeschrittenen, nichtlipomatösen Weichteilsarkomsubtypen (Pazopanib, Regorafenib) eingesetzt [6, 19]. Wenngleich der exakte Wirkmechanismus für diese Multikinaseinhibitoren nicht abschließend geklärt ist, und zuverlässige, selektive Biomarker noch weitgehend fehlen, dürfte der antiangiogenetischen Wirkkomponente eine wesentliche Bedeutung zukommen [19]. Diese Annahme wird ferner dadurch unterstützt, dass viele dieser Medikamente (z. B. Pazopanib oder Sunitinib) auch bei fortgeschrittenen Nierenzellkarzinomen angewandt werden.

Für Angiosarkome oder maligne solitäre fibröse Tumoren (SFT; vormals „Hämangioperizytome") existieren ebenfalls positive Daten betreffend der Verabreichung diverser antiangiogenetischer Inhibitoren. Dazu zählen Multikinaseinhibitoren wie Pazopanib oder Sunitinib, aber auch der monoklonale, antiangiogenetische Antikörper Bevacizumab (Avastin $^{\circledR}$, Roche). Große, prospektive Studien hierfür fehlen aber noch $[1,6$, $19,25]$.

Große Hoffnungen liegen aktuell auf dem kleinmolekularen Inhibitor Larotrectinib (Vitrakvi ${ }^{\circledR}$; Loxo Oncology Inc./ Bayer AG, Leverkusen, Deutschland). Dieser wurde im November 2018 von 


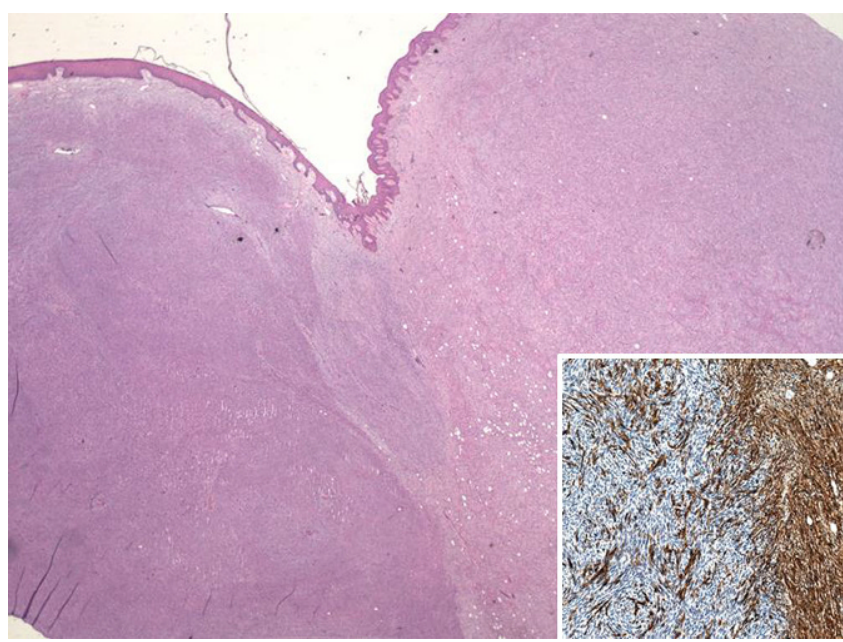

Abb. 5 ॥ Klassischer Teil eines DFSP (Dermatofibrosarcoma protuberans; links) mit abruptem Übergang in den fibrosarkomatösen Anteil des DFSP. Insert: In der Immunhistochemie zeigt sich eine starke CD34-Expression im klassischen Anteil des Tumors, nicht jedoch im fibrosarkomatösen Anteil. Next Generation Sequencing: Nachweis der typischen Fusion COL1A1-PDGFB (COL1A1 "Collagen type I alpha 1 chain", PDGFB "platelet-derived growth factor beta polypeptide“; nicht illustriert). (Mit freundl. Genehmigung, ๑ B. Liegl-Atzwanger, alle Rechte vorbehalten)

der FDA für Erwachsene und Kinder mit soliden Tumoren zugelassen, und weist eine Fusion des „,neurotrophic receptor tyrosin kinase"(NTRK)-Gens auf [23]. $\mathrm{Zu}$ diesen zählen auch mesenchymale Tumoren, wie beispielsweise das infantile Fibrosarkom (• Abb. 5; [23]). Die Zulassung basierte auf drei Phase-Iund -II-Multicenterstudien, in welchen insgesamt $75 \%$ der Tumoren auf diese Therapie ansprachen [23]. Weitere klinische Studien mit NTRK-Inhibitoren laufen [23].

\section{Neue Therapie der aggressiven Fibromatose?}

Kinaseinhibitoren wie Imatinib, $\gamma$-Sekretaseinhibitoren (Nirogacestat, SpringWorks Therapeutics, Stamford, CT, USA; NCT03785964) oder Pazopanib werden aktuell für die Therapie der progredienten bzw. symptomatischen aggressiven Fibromatose vom Desmoidtyp eingesetzt bzw. evaluiert (• Abb. 6; [6]). Darüber hinaus existieren äußerst vielversprechende Daten für die Behandlung der aggressiven Fibromatose mit dem Multikinaseinhibitor Sorafenib [10]. Gounder et al. 2018 berichten im New England Journal of Medicine von einer USamerikanischen doppelblinden, rando-

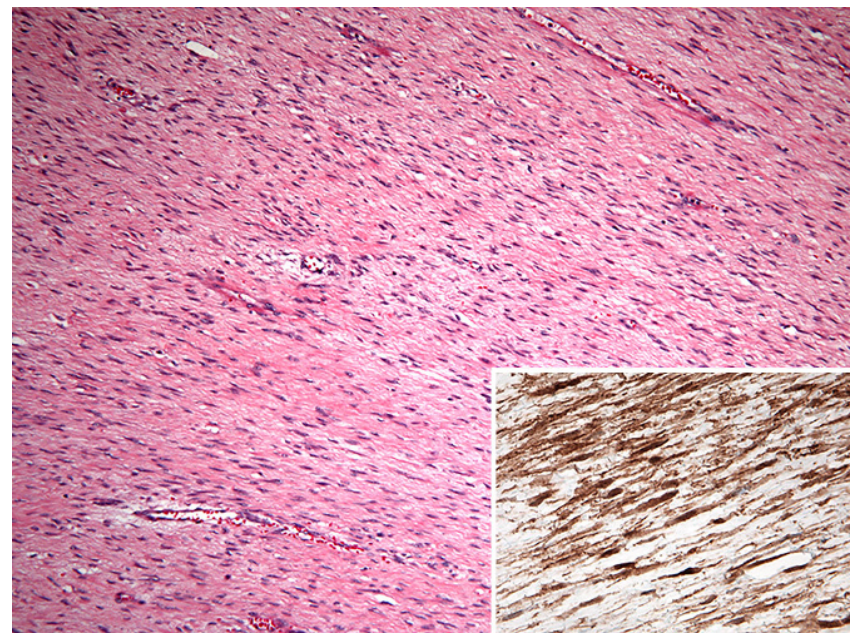

Abb. 6 A Fibromatose vom Desmoidtyp mit charakteristischer nukleärer $\beta$-Catenin-Expression (Insert). (Mit freundl. Genehmigung, @ B. Liegl-Atzwanger, alle Rechte vorbehalten)

\section{Medikamente gegen Riesenzell- tumoren der Sehnenscheiden}

misierten Phase-III-Multicenterstudie. Diese vergleicht insgesamt 87 Patientinnen und Patienten mit progredienter, symptomatischer oder rezidivierender Fibromatose, welche entweder Sorafenib (als 400-mg-Tablette einmal täglich) oder ein Plazebo erhalten hatten [10]. Bei einem durchschnittlichen Follow-up von 27,2 Monaten lag der Prozentsatz an progressionsfreiem 2-Jahres-Überleben bei $81 \%$ in der Sorafenib-Gruppe versus $36 \%$ in der Plazebogruppe. Anders ausgedrückt, hatten Patientinnen und Patienten in der Sorafenib-Gruppe ein um $87 \%$ geringeres Risiko für ein Voranschreiten der Krankheit als jene in der Plazebogruppe [10]. Der exakte Wirkmechanismus, bzw. ob oder inwieweit die Wirkung von Sorafenib mit der bei desmoidartigen Fibromatosen beobachteten $\beta$-Catenin-Mutation $\mathrm{zu}$ sammenhängt, ist noch nicht bekannt [10]. Sofern sich diese Ergebnisse in weiteren (Langzeit-)Studien an größeren Kollektiven bestätigen lassen und die Nebenwirkungen der Therapie von den Patientinnen und Patienten toleriert werden, könnte Sorafenib zu einem fixen Bestandteil im therapeutischen Algorithmus der aggressiven Fibromatose werden.
Auch für Riesenzelltumoren der Sehnenscheiden vom diffusen Typ wird der Einsatz von maßgeschneiderten Therapeutika evaluiert [26]. Diese Tumoren werden grundsätzlich als benigne eingestuft, können jedoch einen aggressiven und rezidivierenden biologischen Verlauf aufweisen, der bis hin zur Amputation führen kann $[18,26]$. Zielstruktur der therapeutischen Ansätze ist in diesem Fall der Monozytenkolonien-stimulierende Faktor ([M-]CSF1; "colony stimulating factor"-1) [26]. Dieser wird bei Riesenzelltumoren der Sehnenscheiden vermehrt exprimiert, vielfach als Ergebnis einer charakteristischen Translokation, in welche das CSF-1-Gen (1p13) involviert ist, zum Teil jedoch auch ohne Vorliegen dieser Translokation aus bisher noch nicht abschließend geklärter Ursache [26]. Da Imatinib unter anderem auch den CSF1-Rezeptor hemmt, wird dieser geläufige Kinaseinhibitor aktuell in der Therapie rezidivierender und anderweitig nicht beherrschbarer Riesenzelltumoren der Sehnenscheide vom diffusen Typ evaluiert $[6,26]$. Darüber hinaus bestehen Ansätze, Inhibitoren zu entwickeln, welche eine optimale Hemmwirkung auf den CSF-1-Rezeptor (CSF-1R) ausüben [26]. 
Bisher kein Benefit histologiespezifischer Chemotherapien

Darüber hinaus existieren Ansätze, histologiespezifische neoadjuvante Chemotherapien bei "high-grade" Weichteilsarkomen zu verabreichen. Diese Idee fußt auf der Beobachtung, dass die Gabe des ursprünglich aus Meereslebewesen gewonnenen Chemotherapeutikums Trabectedin (Yondelis ${ }^{\circledR}$, PharmaMar, Madrid, Spanien) in der Subgruppe der myxoiden Liposarkome, verglichen mit anderen Entitäten, ein besonders gutes Ansprechen gezeigt hat $[3,6]$. Erste Ergebnisse einer randomisierten multizentrischen Studie lassen bislang für die Mehrzahl der untersuchten histologischen Subtypen jedoch keinen Benefit solcher "individualisierten" Chemotherapien gegenüber Standardchemotherapien bei Weichteilsarkomen erkennen [6, 11]. Trabectedin lieferte bei myxoiden Liposarkomen zumindest äquivalente Ergebnisse, jedoch keinen eindeutigen Benefit $[6,11]$. Wenngleich diese Studie einige Limitationen aufweist, wie beispielsweise ein vergleichsweise kurzes Follow-up, hat sie die Erwartungen an „personalisierte Chemotherapien“ jedenfalls merklich gedämpft [11]. Ebenso wenig konnten Kombinationstherapien von Chemotherapeutika und Kinaseinhibitoren die in sie gesetzten Erwartungen bislang erfüllen: die Kombination von Olaratumab (Lartruvo ${ }^{\circledR}$, Eli Lilly, Indianapolis, IN, USA; ein gegen PDGFRa gerichteter monoklonaler Antikörper) mit dem Chemotherapeutikum Doxorubicin zeigte initial in einer Phase-IIStudie ein verbessertes Gesamtüberleben $[6,28]$. Eine konsekutive Phase-IIIStudie konnte diese Ergebnisse jedoch nicht bestätigen, sodass die weitere Entwicklung dieses Antikörpers seitens des Pharmaunternehmens gestoppt wurde [2].

\section{Ausblick}

Die angeführten Beispiele illustrieren vielversprechende Ansätze zum Einsatz personalisierter Therapeutika bei Tumoren des Stütz- und Bewegungsapparates. Dem stehen ernüchternde Ergebnisse großer, randomisierter Stu- dien gegenüber, welche Hoffnungen in histologiespezifische Chemotherapien oder synergistische Effekte von Chemotherapeutika und Rezeptorblockern vorerst nicht erfüllen konnten. Es wird zunehmend klarer, dass die Tumorentstehung ein komplexer Mechanismus ist, welchen man nicht mit der Hemmung eines einzelnen Targets nachhaltig und vollständig unterbinden kann $[5,21]$. Dazu kommt, dass viele der zur Tumorentstehung führenden Mechanismen noch nicht abschließend geklärt sind. Nachgewiesene genetische Veränderung stellen oft nur die Spitze des Eisberges dar, den es tiefgehender zu ergründen gilt, um daraus Therapieansätze ableiten zu können [5, 19, 21]. Intratumorale Heterogenitäten, Tumorplastizität und die Entwicklung von Resistenzmechanismen bedingen darüber hinaus, dass die Wirkung personalisierter Therapeutika meist nur auf wenige Monate beschränkt ist [5]. Darüber hinaus sind die Nebenwirkungen, insbesondere von Multikinaseinhibitoren, oft nicht unerheblich [13], und die Behandlungskosten hoch. Dennoch haben die vergangenen Jahre viele neue Ansätze erbracht, und unser Verständnis von Tumorentstehung und -subklassifizierung nachhaltig verändert $[19,20]$.

\section{》) Viele der zur Tumorentste- hung führenden Mechanismen sind noch nicht abschließend geklärt}

Spezielle Forschungsansätze und Studiendesigns, wie beispielsweise BasketStudien (englisch "trials") tragen diesem Umstand bereits Rechnung. Sie untersuchen das Ansprechen unterschiedlicher Erkrankungen, welche dieselbe genetische Veränderung aufweisen, auf ein gegen diese genetische Veränderung gerichtetes Medikament [19]. Dadurch können auch ultraseltene Erkrankungen, wie Sarkome und ihre Subgruppen, in diese Studien eingeschleust und neuen Behandlungen zugänglich gemacht werden.

Wenngleich wir von der Ehrlich-Version eines „Magic Bullet“ noch weit ent- fernt sind, stehen wir inmitten einer Entwicklung, welche das Potenzial hat, unser Krankheitsverständnis sowie die Behandlungsmöglichkeiten von Sarkomen nachhaltig zu verändern.

\section{Fazit für die Praxis}

- Die Molekularpathologie ist fixer Bestandteil der Routinediagnostik von Tumoren des Stütz- und Bewegungsapparates.

- Für Knochensarkome existieren noch keine routinemäßig einsetzbaren personalisierten Therapeutika. Denosumab ist als symptomatische Behandlung von Riesenzelltumoren zu werten.

- Tyrosinkinaseinhibitoren (z. B. Imatinib, Pazopanib, Sorafenib oder Larotrectinib) und monoklonale Antikörper (z. B. Bevacizumab) werden in der Therapie von Tumoren des Stützund Bewegungsapparates, insbesondere der Weichteile, evaluiert.

- Große, randomisierte Studien konnten Erwartungen in histologiespezifische Chemotherapien bzw. synergistische Effekte von Chemotherapeutika und Rezeptorblockern vorerst nicht erfüllen.

- Ein besseres Verständnis der Auswirkungen molekularpathologischer Veränderungen ist nötig, um treffsichere Behandlungen entwickeln zu können.

- Klinische Studienansätze müssen effizienter werden und der zunehmenden Subtypisierung von Sarkomen Rechnung tragen.

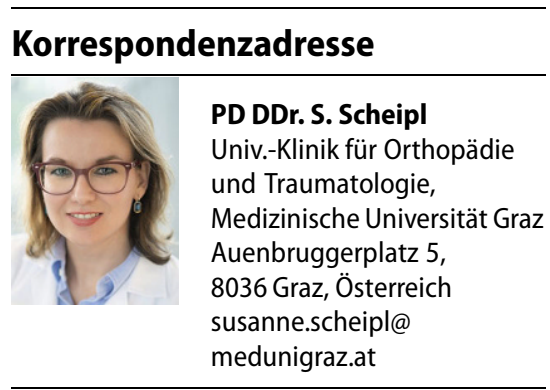

Funding. Open access funding provided by Medical University of Graz. 


\section{Einhaltung ethischer Richtlinien}

Interessenkonflikt. S. Scheipl, B. Liegl-Atzwanger, J. Szkandera, B. Rinner, C. Viertler, J. Friesenbichler, M. Bergovec und A. Leithner geben an, dass kein Interessenkonflikt besteht.

Für diesen Beitrag wurden von den Autoren keine Studien an Menschen oder Tieren durchgeführt. Für die aufgeführten Studien gelten die jeweils dort angegebenen ethischen Richtlinien.

Open Access. Dieser Artikel wird unter der Creative Commons Namensnennung 4.0 International Lizenz (http://creativecommons.org/licenses/by/4.0/deed. de) veröffentlicht, welche die Nutzung, Vervielfältigung, Bearbeitung, Verbreitung und Wiedergabe in jeglichem Medium und Format erlaubt, sofern Sie den/die ursprünglichen Autor(en) und die Quelle ordnungsgemäßnennen, einen Linkzur Creative Commons Lizenz beifügen und angeben, ob Änderungen vorgenommen wurden

\section{Literatur}

1. Agulnik M, Yarber JL, Okuno SH et al (2013) An open-label, multicenter, phase II study of bevacizumab for the treatment of angiosarcoma and epithelioid hemangioendotheliomas. Ann Oncol 24:257-263

2. Anonymous (2019) Olaratumab for STS Disappoints in Phase III. Cancer Discov 9:312-313

3. Assi T, Kattan J, El Rassy E et al (2019) A comprehensive review of the current evidence for trabectedin in advanced myxoid liposarcoma. Cancer Treat Rev 72:37-44

4. Behjati S, Tarpey PS, Presneau N et al (2013) Distinct $\mathrm{H} 3 \mathrm{~F} 3 \mathrm{~A}$ and $\mathrm{H} 3 \mathrm{~F} 3 \mathrm{~B}$ driver mutations define chondroblastoma and giant cell tumor of bone. Nat Genet 45:1479-1482

5. Burrell RA, Swanton C (2014) Tumour heterogeneity and the evolution of polyclonal drug resistance. Mol Oncol 8:1095-1111

6. Casali PG, Abecassis N, Bauer S et al (2018) Soft tissue and visceral sarcomas: ESMO-EURACANClinical Practice Guidelines for diagnosis, treatment and follow-up. Ann Oncol 29:iv51-iv67

7. Casali PG, Bielack S, Abecassis N et al (2018) Bone sarcomas: ESMO-PaedCan-EURACANClinical Practice Guidelines for diagnosis, treatment and follow-up. Ann Oncol 29:iv79-iv95

8. D'angelo SP, Melchiori L, Merchant MS et al (2018) Antitumor activity associated with prolonged persistence of adoptively transferred NY-ESO-1 (c259)T cells in synovial sarcoma. Cancer Discov 8:944-957

9. Erkizan HV, Kong Y, Merchant M et al (2009) A small molecule blocking oncogenic protein EWS-FLI1 interaction with RNA helicase $A$ inhibits growth of Ewing's sarcoma. Nat Med 15:750-756

10. Gounder MM, Mahoney MR, Van Tine BA et al (2018) Sorafenib for advanced and refractory Desmoid tumors. N Engl J Med 379:2417-2428

11. Gronchi A, Ferrari S, Quagliuolo V et al (2017) Histotype-tailored neoadjuvant chemotherapy versus standard chemotherapy in patients with high-risk soft-tissue sarcomas (ISG-STS 1001): an international, open-label, randomised, controlled, phase 3, multicentretrial.LancetOncol 18:812-822
12. Guilhot F (2004) Indications for imatinib mesylate therapy and clinical management. Oncologist 9:271-281

13. Hartmann JT, HaapM, KoppHGetal (2009) Tyrosine kinase inhibitors-a review on pharmacology, metabolism and side effects. Curr Drug Metab 10:470-481

14. Heery CR, Singh BH, Rauckhorst M et al (2015) Phase I trial of a yeast-based therapeutic cancer vaccine (GI-6301) targeting the transcription factor Brachyury. Cancer Immunol Res 3:1248-1256

15. Hogendoorn PCW, Bovée JVMG, Nielsen GP (2013) Chondrosarcoma (grades I-III), including primary and secondary variants and periosteal chondrosarcoma. In: Fletcher CDM, Bridge JA, Hogendoorn PCW, Mertens F (Hrsg) WHO Classification of Tumours of Soft Tissue and Bone. IARC, Lyon, S264-268

16. Kovac M, Blattmann C, Ribi S et al (2015) Exome sequencing of osteosarcoma reveals mutation signatures reminiscent of BRCA deficiency. Nat Commun 6:8940

17. Li X, Seebacher NA, Hornicek FJ et al (2018) Application of liquid biopsy in bone and soft tissue sarcomas: present and future. Cancer Lett 439:66-77

18. Mastboom MJL, Verspoor FGM, Gelderblom H et al (2017) Limb amputation after multiple treatments of Tenosynovial giant cell tumour: series of 4 Dutch cases. Case Rep Orthop 2017:7402570

19. Nakano K, Takahashi S (2018) Current molecular targeted therapies for bone and soft tissue sarcomas. Int J Mol Sci 19:739. https://doi.org/10 3390/ijms19030739

20. Nakano K, Takahashi S (2018) Translocationrelated sarcomas. Int J Mol Sci 19:3784. https://doi. org/10.3390/ijms19123784

21. Scannell JW, Blanckley A, Boldon $\mathrm{H}$ et al (2012) Diagnosing the decline in pharmaceutical $R \& D$ efficiency. Nat Rev Drug Discov 11:191-200

22. Schaefer IM, Hornick JL, Bovee J (2018) The role of metabolic enzymes in mesenchymal tumors and tumor syndromes: genetics, pathology, and molecular mechanisms. Lab Invest 98:414-426

23. Scott LJ (2019) Larotrectinib: first global approval. Drugs 79:201-206

24. Sheffield NC, Pierron G, Klughammer J et al (2017) DNA methylation heterogeneity defines a disease spectrum in Ewing sarcoma. Nat Med 23:386-395

25. Spagnuolo RD, Brich S, Bozzi F et al (2016) Sunitinib-induced morpho-functional changes and drug effectiveness in malignant solitary fibrous tumours. Oncotarget 7:45015-45026

26. Stephan SR, Shallop B, Lackman R et al (2016) Pigmented Villonodular Synovitis: a comprehensive review and proposed treatment algorithm. JBJS Rev 4. https://doi.org/10.2106/jbjs.rvw.15.00086

27. Strebhardt K, Ullrich A (2008) Paul Ehrlich's magic bullet concept: 100 years of progress. Nat Rev Cancer 8:473-480

28. Tap WD, Jones RL, Van Tine BA et al (2016) Olaratumab and doxorubicin versus doxorubicin alone for treatment of soft-tissue sarcoma: an open-label phase $1 \mathrm{~b}$ and randomised phase 2 trial. Lancet 388:488-497

29. Toporkiewicz M, Meissner J, Matusewicz L et al (2015) Towardamagic or imaginarybullet? Ligands for drug targeting to cancer cells: principles, hopes, and challenges. Int J Nanomedicine 10:1399-1414

30. Tsafou K, Katschnig AM, Radic-Sarikas B et al (2018) Identifying the druggable interactome of EWS-FLI1 reveals MCL-1 dependent differential sensitivities of Ewing sarcoma cells to apoptosis inducers. Oncotarget 9:31018-31031
Kalkschulter: Schmerzen gezielt mit Stoßwellen lindern

Die Erkrankung wird meistens erst recht spät diagnostiziert, denn solange die Kalkablagerungen klein sind, verursachen sie keine Symptome. Warum sich die Kalkansammlungen bilden, ist noch nicht endgültig geklärt. Eine Ursache könnte eine Mangeldurchblutung und damit ein Sauerstoffmangel in den Schultersehnen sein. Ein therapeutischer Ansatz ist deshalb die Aktivierung des Stoffwechsels durch gezielte Bewegung. Bei Bedarf verordnet der Arzt zudem noch Medikamente gegen Schmerzen oder Entzündungen. Manchmal setzen auch Physiotherapeuten therapeutischen Ultraschall unterstützend zur Schmerzbehandlung ein.

Bei großen Kalkansammlungen wird den Patienten manchmal zur Operation geraten. Eine schonende Alternative dazu kann jedoch die Stoßwellentherapie sein. Der Nutzen der Behandlung in Bezug auf eine Besserung der Symptome und Verkleinerung der Kalkdepots ist inzwischen in vielen Studien nachgewiesen.

Bei der Therapie der Kalkschulter richtet der Arzt fokussierte Stoßwellen gezielt auf das Kalkdepot in der Schultersehne. Das Gewebe um die Kalkansammlung wird nicht beeinträchtigt

Ein bis drei Behandlungen sind in der Regel ausreichend. Die Nebenwirkungen sind gering, die Patienten werden durch dieses schonende Verfahren häufig wieder schmerzfrei und in der Schulter beweglich. Die Krankheitsdauer wird verkürzt und eine Operation in der Regel vermeidbar.

Quelle: http://www.degum.de 